Research Paper

\title{
Programmed Death Ligand 1 (PD-L1) Expression in Primary Angiosarcoma
}

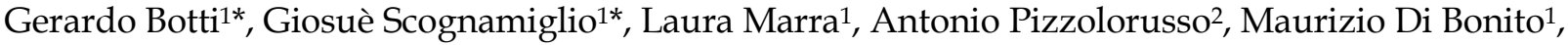 \\ Rossella De Cecio ${ }^{1}$, Monica Cantile ${ }^{1 凶}$, Annarosaria De Chiara ${ }^{1}$ \\ 1. Pathology Unit, Istituto Nazionale Tumori Fondazione "G. Pascale", via Mariano Semmola, 80131 Napoli, Italy; \\ 2. Department of Muscle-skeletal Oncology, Istituto Nazionale Tumori Fondazione "G. Pascale", via Mariano Semmola, 80131 Napoli, Italy. \\ * Equal contributors \\ $\triangle$ Corresponding author: Monica Cantile: Pathology Unit, Istituto Nazionale Tumori Fondazione “G. Pascale”, via Mariano Semmola, 80131 Napoli, Italy \\ TEL.+390815903745; FAX. +390815903718; e-mail: m.cantile@istitutotumori.na.it \\ ( $)$ Ivyspring International Publisher. This is an open access article distributed under the terms of the Creative Commons Attribution (CC BY-NC) license \\ (https://creativecommons.org/licenses/by-nc/4.0/). See http://ivyspring.com/terms for full terms and conditions.
}

Received: 2017.01.05; Accepted: 2017.03.31; Published: 2017.09.15

\begin{abstract}
Angiosarcomas are rare malignant endothelial-cell tumors of vascular or lymphatic origin, and are among the most aggressive subtypes of soft-tissue sarcomas. The prognosis is poor and treatment is challenging in many cases. PD-1/PD-Ll pathway plays a critical role in immune escape of tumor cells. Recent studies described that PD-L1 is widely expressed in various types of cancer, providing the basis for the development of PDI/PD-L1 antibodies as anti-cancer immunotherapy. Despite the well-known potential of PD-L1 as prognostic and predictive biomarker, only few studies described its IHC expression in cancer subtypes for the extreme difficulty in developing standard protocol with the different antibody clones available.

We analyzed the IHC expression of PD-LI on a series of angiosarcomas at different body location, showing its aberrant expression in about $66 \%$ of samples with no relation with prognosis. Our study allowed us to correctly define PD-LI staining in angiosarcoma tumor tissues with final purpose to stratify patients for immune checkpoint inhibitors therapies.
\end{abstract}

Key words: angiosarcoma, PD-L1, IHC evaluation.

\section{Introduction}

Angiosarcomas are rare malignant vascular tumors, which account for approximately $2 \%$ of all soft tissue sarcomas. It is characterized by rapidly proliferating, extensively infiltrating atypical cells derived from blood vessels and lining irregular blood-filled spaces [1].

Angiosarcomas can arise at different sites and in different organs with distinct features, more frequently in skin and soft tissue, but also in the liver, breast, spleen, bone, or heart.

The most common form of angiosarcoma is cutaneous angiosarcoma, with a higher incidence in elderly patients [2], followed by soft tissue angiosarcomas with a very aggressive clinical behaviour [1], while primary mammary angiosarcoma, affecting a mainly younger females is very uncommon [3].

Several studies suggested that angiosarcomas had a very poor prognosis, with 5-year survival of only $10-15 \%$. This highly aggressive tumour spreads widely, recurs locally and metastasizes early [1].

Surgery is the mainstay of treatment, but the high frequency of local recurrence of this strategy is discouraging. Radiotherapy was generally performed in cases of widely spread and unresectable tumours, but the outcomes were usually unsatisfactory $[4,5]$. Therefore, further adjuvant or supportive therapy is necessary for the treatment of angiosarcoma. Recently, chemotherapy and immunotherapy using recombinant interleukin-2 (rIL-2) have been studied 
as potential treatments [6]. However, the optimal treatment for these tumours has not been clearly established.

PD-L1 is a $40 \mathrm{kDa}$ transmembrane protein that is expressed on a wide variety of normal tissues including natural killer cells, macrophages, myeloid dendritic cells, B cells, epithelial cells, and vascular endothelial cells [7]. Recently, several studies showed that PD-1/PD-L1 pathway may have a key role in the interaction of tumor cells with host immune response, and tumor cells PD-L1 expression may serve as a mechanism of adaptive immune resistance [8]. Many human cancers have been shown to express PD-L1 in most of cases, and its expression was also correlated with a poor prognosis, highlighting that it can represents a potential prognostic and predictive biomarker in human solid tumors [9-13]. In support of these experimental evidences, early-phase trials using monoclonal antibodies targeting PD-1 or PD-L1 revealed a real efficacy in patients with refractory tumors [14].

However, recent reports revealed that the expression of the PD-L1 on tumor cells is not uniform, because of different antibodies clones, with variable specificity, often doubtful topographical localization and not well defined score.

The purpose of this study was to analyze the immunohistochemical expression of PD-L1 to define adequate evaluation criteria, and investigate its prognostic role in angiosarcomas.

\section{Materials and Methods}

\section{Patient selection}

From 2006 to 2015, 24 Formalin Fixed Paraffin Embedded (FFPE) samples from angiosarcoma patients were retrieved from the Department of Pathology, at the National Cancer Institute "Giovanni Pascale Foundation" of Naples, Italy. All selected cases were primary angiosarcoma. We excluded secondary angiosarcoma originating as consequences of breast radiotherapy.

Medical records for all cases were reviewed for clinical information. The following clinical and pathological parameters were evaluated for each tumor included in the study: patient age at initial diagnosis, tumour size, histologic features, tumour differentiation, type of surgery (for tumour removal) and clinical outcome.

In addition to H\&E evaluation, all specimens were characterized for all routinely diagnostic immunophenotypic parameters.

\section{Immunohistochemical analysis}

Immunohistochemical staining for PD-L1 was performed on 5 - $\mu$ m-thick sections obtained from formalin-fixed, paraffin-embedded tissue of the selected cases. Prepared slides were incubated for 12 minutes at $110^{\circ} \mathrm{C}$ in Cell Conditioning Solution (Ventana medical Systems [Cat \#: 950-124], Tucson, Arizona, USA), using a commercial steamer as the heat source (Biocare Medical, Decloaking Chamber DC12, Pike Lane Concord CA, USA). After cooling for 15 minutes, manual staining was performed. Following a $5 \mathrm{~min}$ incubation with a peroxidase blocking reagent (DAKO \#SM801, Glostrup, Denmark, UE) followed by two, 5 min rinses in Bond Wash Solution (Leica, \#AR9590). Protein blocking reagents were applied for $5 \mathrm{~min}$ with a protein serum block (1\% goat serum (Abcam Cambridge Science Park Milton Road, Cambridge, UK), 4\% BSA in PBS. Blocking reagents were removed without rinsing before adding primary antibody. Primary anti-PDL-1 antibody clone SP-142 (Spring Bioscience [\#M4420] Koll Center Pkwy, Pleasanton, CA, USA) was diluted in one commercial primary antibody diluent (Bond primary antibody diluent, \# AR9352, Leica Biosystems, Newcastle, UK) at a concentration of 3,75 $\mu \mathrm{g} / \mathrm{ml}$. Slides were incubated with the antibody for 1 hour at room temperature, followed by two steps of 5 min in Bond Wash Solution. The Goat Anti-Rabbit + HRP (horseradish peroxidase) visualization reagent (DAKO, Cat \# SK001, Glostrup, Denmark, UE), which is biotin-independent and reduces the potential for background or nonspecific staining from endogenous biotin, was used for primary antibody detection. After two, 5 min rinses in Bond Wash Solution the secondary antibody was incubate for 35-minute. Follow incubation with steady plus DAB (3,3'-diaminobenzidine) kit (ABCAM, \#AB103723,). The slides were rinses for 5 in distillated water. The slides were counterstained with hematoxylin for 30 second, rinsed in distilled water, dehydrated in an ethanol series $(30 \%, 70 \%, 100 \%)$ and four changes of $100 \%$ xylene, and permanently sealed with coverslips in automatic (DAKO \#CS100, Glostrup, Denmark, UE).

\section{Immunohistochemistry evaluation}

Antigen expression was evaluated independently by two pathologists (ADC and MDB) using a light microscopy. For each sample, at least five fields (inside the tumor and in the peripheral areas) and $>500$ cells were analysed. Using a semi-quantitative scoring system microscopically and referring to each antigen scoring method in other studies, each observer evaluated the intensity, extent and subcellular distribution.

For TIL detection, lymphocytes were quantified by manual expression scoring, considering as tumor infiltrating (TIL) if they were located within 1 
high-power field (HPF; $\times 40)$ of carcinoma cells. An immunohistochemistry characterization of immune cells was performed by CD3 (for T-cells), CD20 (for B-cells) and CD163 (for macrophages).

For PD-L1 assessment we considered both a qualitative and a quantitative parameter. For the qualitative criteria we considered i) the immunoreactivity of membrane: "absent" (negative samples), "incomplete" or "complete" (positive samples); and ii) the intensity of the reaction at the membrane level: "mild", "moderate" and "intense". For the quantitative criteria we considered positive samples when PD-L1 staining is detected in $\geq 5 \%$ of tumor cells.

\section{Statistical analysis}

The association between PD-L1 expression with clinical-pathological parameters was conducted using Fisher's Exact test determining whether a relationship exists between the different variables included in the study. The level of significance was defined as $\mathbf{P}<0.05$. Overall survival (OS) and disease-free survival (DFS) curves were calculated using the Kaplan-Meier method. All the statistical analyses were carried out using the Statistical Package for Social Science v. 20 software (SPSS Inc., Chicago, IL, USA).

OS was defined as the time from diagnosis (first biopsy) to death by any cause or until the most recent follow-up. DFS was measured as the time from diagnosis to the occurrence of progression, relapse after complete remission, or death from any cause.

\section{Results}

\section{Clinical-pathological features of angiosarcoma patients}

In our cohort, we have included 24 primary angiosarcoma samples from different body locations.

The age of patients ranged from 23-91 years, with an average age of 58 years. Females were 14/24 while males were 10/24. The prevalent sites of primary lesion were breast $(7 / 24)$, followed by soft tissues $(5 / 24)$, bone $(4 / 24)$, skin $(4 / 24)$, and visceral $(4 / 24)$. Regarding tumor grading and histological differentiation, 12/24 were classified as low grade/well differentiated tumors, and $12 / 24$ as high grade/poor differentiated tumors. All information is summarized in Table 1.

\section{PDLI expression in primary angiosarcoma samples and relation with clinical- pathological features and prognosis of patients}

PD-L1 was detected in 16/24 (66\%) of angiorsarcoma samples, with a heterogeneous expression of PD-L1 on tumor cells.
The incomplete (Figure 1a, 1b) and complete (Figure 1c, 1d) immunoreactivity of membrane was equally distributed in our case series, with a prevalent mild intensity of reaction, and with or without cytoplasmic positivity (Figure 1e, 1f) associated with a membrane staining.

In most of the analyzed samples the presence of infiltrating lymphocyte (TIL) was poor, only in 3/20 $(15 \%)$ it was consistent. TIL component showed a PD-L1 positive staining also in negative tumor samples (Figure 2a, 2b).

Based on statistical elaboration of PD-L1 protein expression analysis with the clinical-pathological parameters in angiosarcomas, we showed no statistically significance with age, gender and site location while a strong statistical association was detected with tumor differentiation ( $p$-value $=0.027$ ). PD-L1 appeared overexpressed in high grade/poor differentiated tumors, and variably expressed in low grade/well differentiated angiosarcomas. All data are schematized in Table 1.

Regarding the relation with patients survival, we showed only a weak trend of statistical association with DFS (0.118) (metastatic patients have a prevalent expression of PD-L1), while no statistically significance was detected with Overall survival $(\mathrm{p}$-value $=0.726)$.

Kaplan-Meier curves relative to DFS and OS are illustrated in Figure 3.

Table 1. Statistical association of PD-L1 tumor expression with clinic pathological features of angiosarcoma patients.

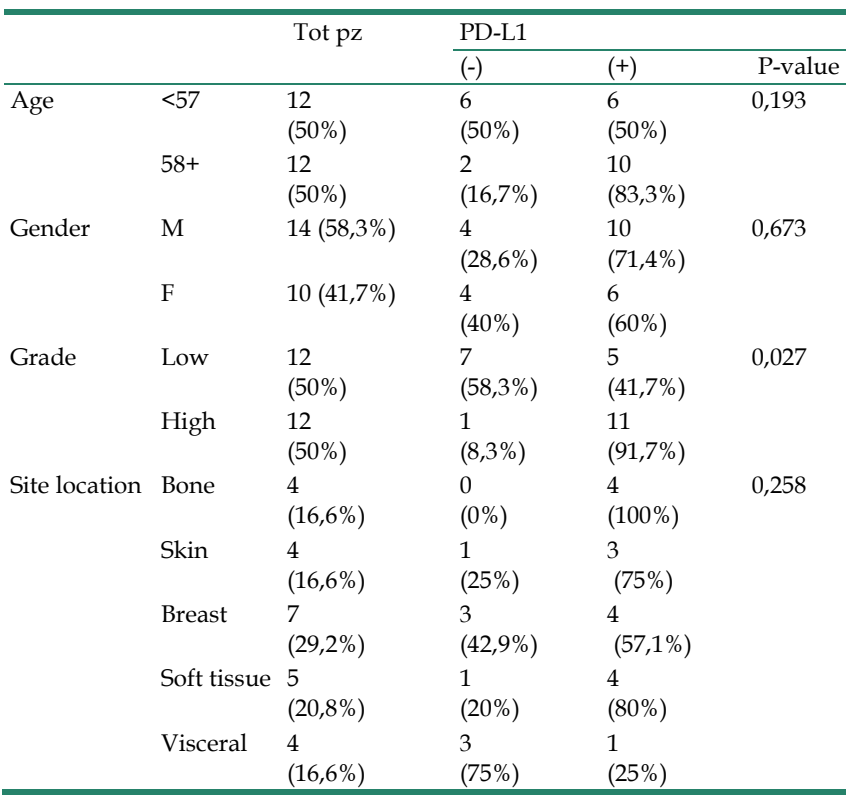




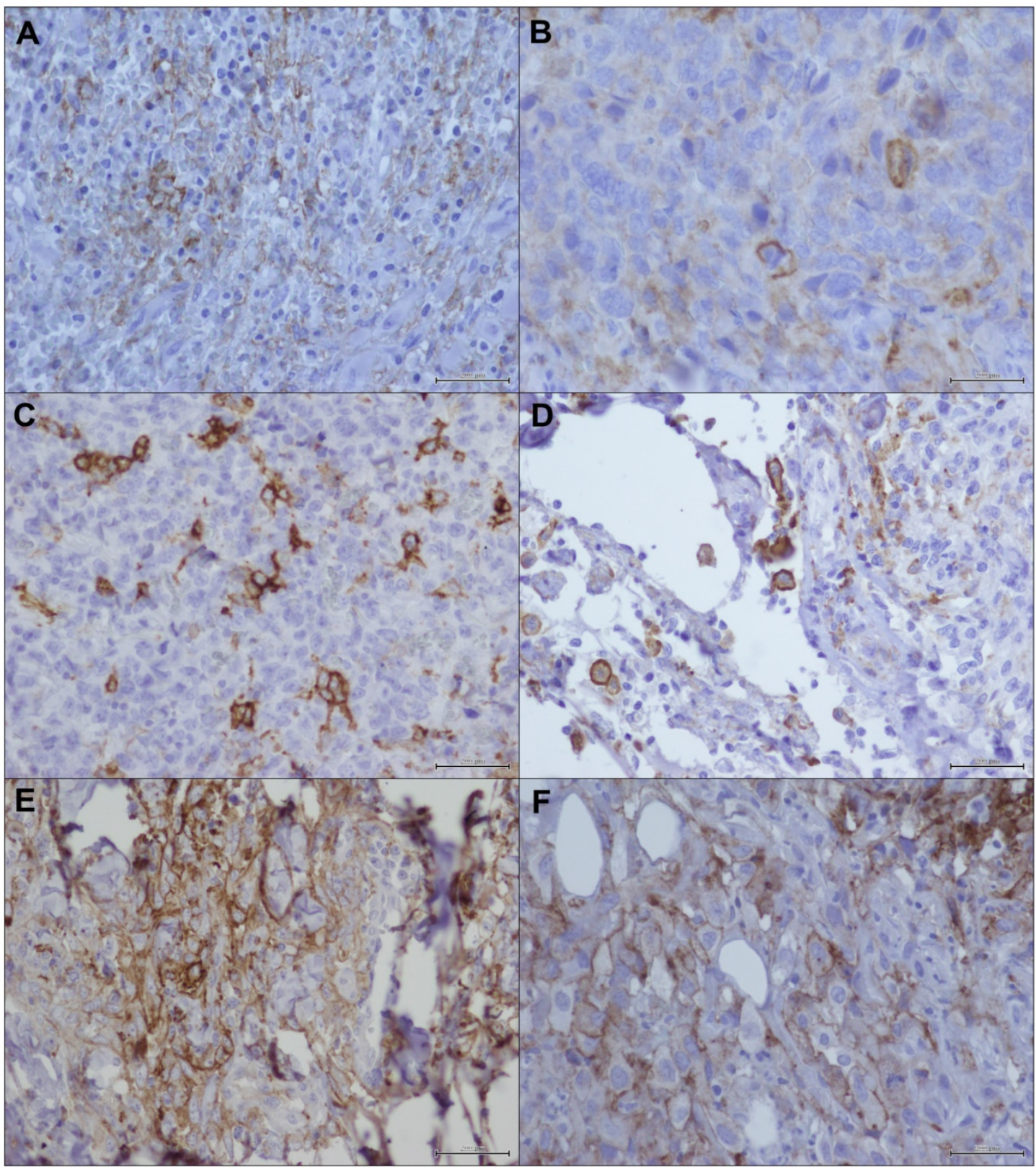

Figure 1. PD-L1 immunostaining in angiosarcoma: a) incomplete immunoreactivity of membrane (20X); b) detail of incomplete immunoreactivity of membrane (40X); c) complete immunoreactivity of membrane (20X); d) detail of complete immunoreactivity of membrane (40X); e) immunoreactivity of membrane with cytoplasmic positivity (20X); f) detail of immunoreactivity of membrane with cytoplasmic positivity (40X).

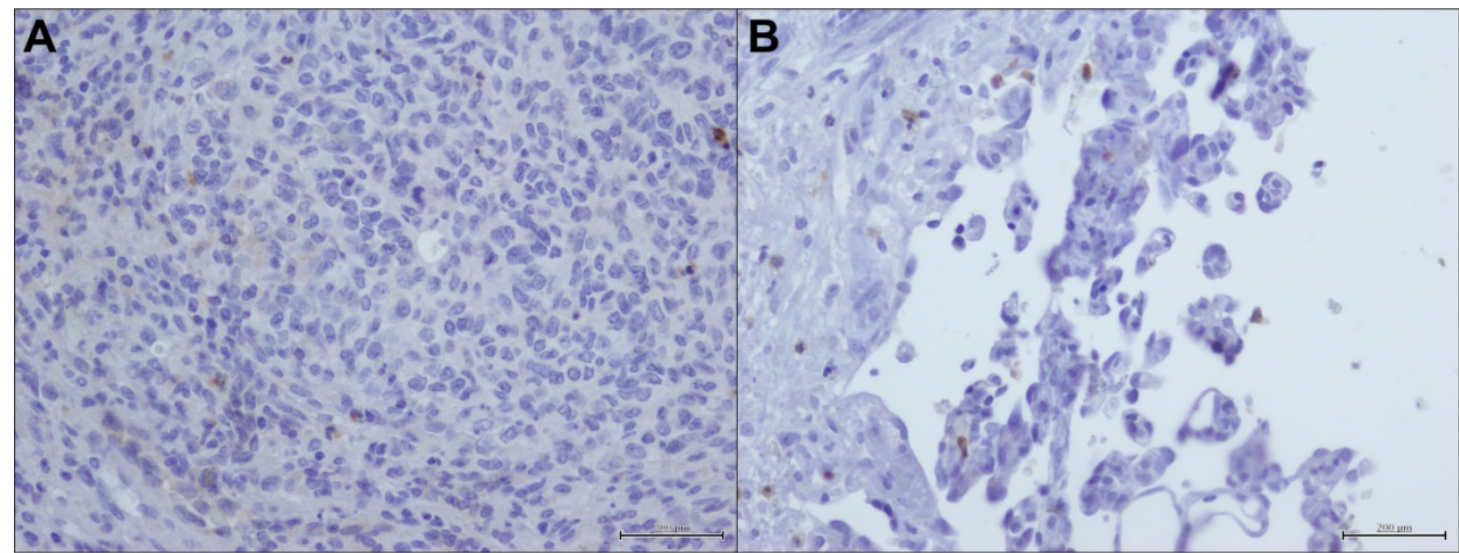

Figure 2. PD-L1 immunostaining in immune cells within angiosarcoma samples (20X). 


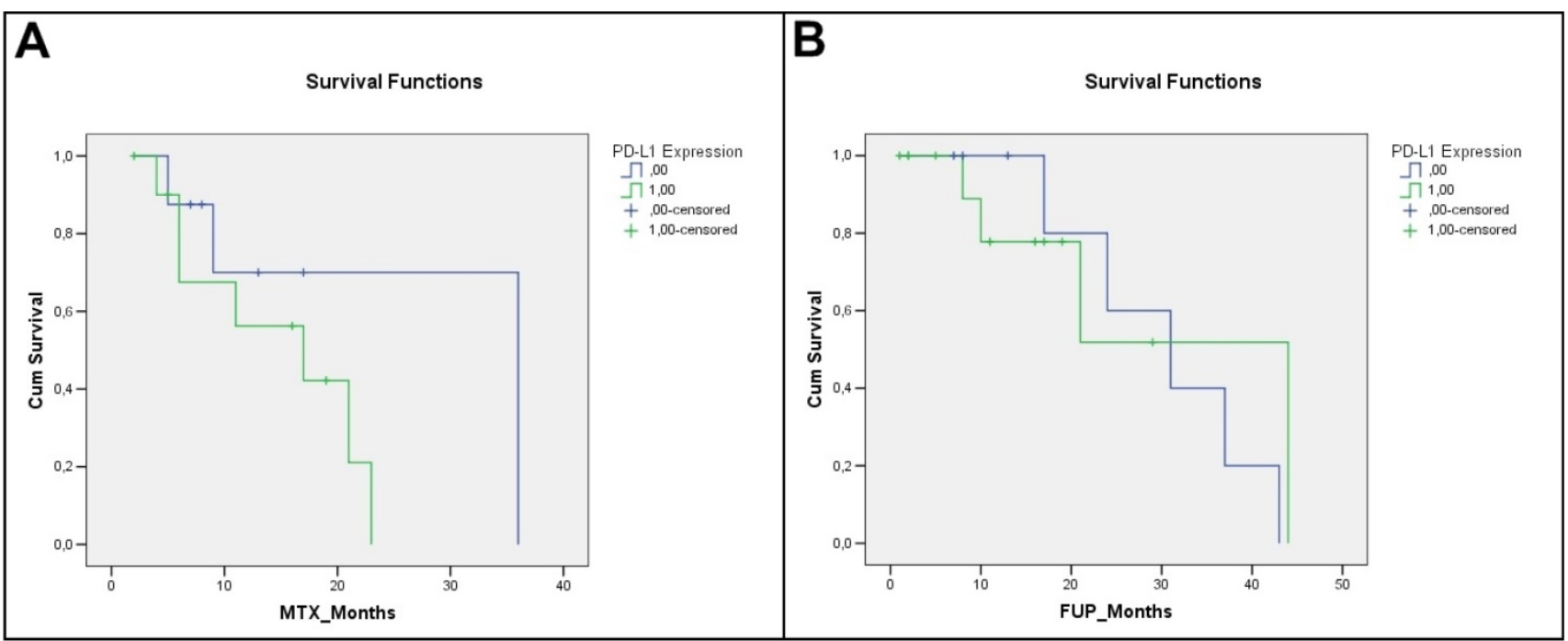

Figure 3. Angiosarcoma patients Kaplan-Meier curves: a) Disease Free Survival (DFS) $(p=0,118)$; b) Overall Survival (OS) $(p=0,726)$.

\section{Discussion}

Angiosarcomas are rare malignant mesenchymal tumours, showing a wide range of differentiation from highly differentiated tumours, which resemble hemangiomas, to poorly differentiated anaplastic lesions [1].

Primary Angiosarcoma had a very poor prognosis with a high rate of metastasis involving most commonly lungs, liver, regional lymph nodes and bone.

Cutaneous angiosarcoma is the most common form of angiosarcoma [2], with a higher incidence in elderly patients as well as Soft Tissue Angiosarcomas [1]. Primary angiosarcoma of the Breast is the most common mesenchymal tumour of the breast while angiosarcoma of the Gastrointestinal Tract is quite rare. Both lesions, in the most of cases, are followed to a radiation therapy for other malignant and benign diseases [15].

Angiosarcoma is a tumor with a very poor prognosis, also because of known molecular alterations and consequently of specific therapeutic targets. Therefore, it is treated mainly with surgery followed by chemotherapy and/or radiation therapy.

Several drugs directed against PD-1/PD-L1 axis are now undergoing clinical trials in various tumor diseases [16], but PD-L1 role as prognostic and predictive biomarker, among various studies, is very moot [17-23] also because the different assays for IHC staining and different cutoff values assigned.

In this study we assessed the immunohistochemical expression of PD-L1 mainly to clarify the potential role in the progression of angiosarcoma, but also to define a correct IHC staining.
A case series of 24 angiosarcomas from different body localization were enrolled into this study. Our results showed a heterogeneous expression of PD-L1 on tumor cells.

For its assessment and evaluation, not yet described in the literature, we defined both qualitative, considering the immunoreactivity of membrane and the intensity of the reaction at the membrane level, and quantitative parameters, considering the percentage of positive tumor cells $\geq$ $5 \%$.

PD-L1 was detected in about $66 \%$ of angiosarcoma samples with a prevalent immunoreactivity of membrane. Only in few cases a mild cytoplasmic associated with membrane staining was present.

The data available in the literature related to the expression of PD-L1 are not uniform, because of the different antibodies clones used, with variable specificity, often doubtful topographical localization and not well defined score. Moreover, in the most of studies in which PD-L1 expression was realized by immunohistochemistry, a consistent cytoplasmic expression was detected, without clarify and define the real value of this positivity in tumor cells [24].

Whereas for other malignancies the prognostic value of PD-L1 is abundantly described in the literature, also for the therapeutic framing of the patients, for the soft tissue tumors this role has not been yet well defined. In a single study PD-L1 IHC expression was described in several histological subtypes of sarcomas [25]. However, the limitation of this study was that PD-L1 expression was analyzed on 20 different histological types, with a few number of specimens for each tumor types without defining its prognostic value. In this study D'Angelo SP et al. 
described the PDL-1 expression in the all three angiosarcoma cases and also in TIL inside tumors [25].

In our series, PD-L1 showed only a strong statistical association with tumor differentiation. PD-L1 was overexpressed in high grade/poor differentiated tumors, and variably expressed in low grade/well differentiated angiosarcomas. No significant statistical association was detected with DFS and OS, highlighting that PD-L1 would not seem to be a prognostic marker in this tumor disease.

Actually the prognostic value of PD-L1 in solid tumors is much debated and often conflicting data were described in the literature.

Although most of the findings associated PD-L1 expression with a poor prognosis [26, 27], several studies assigned an opposite value to its expression.

Schalper KA et al. quantized in situ PD-L1 mRNA levels in a large case series of stage I-III breast carcinomas showing that its expression was associated with longer recurrence-free survival [17].

Moreover, another immunohistochemical study showed that PD-L1 positive patients had a significantly greater disease-control rate, in association with longer progression-free survival after EGFR-tyrosine kinase inhibitor therapy and overall survival in EGFR mutation positive Lung adenocarcinoma [22].

More recently Yang et al. described that tumor PD-L1 expression and increased CD4+T cell infiltrations in the tumor stroma were independent predictors of better overall survival in stage I pulmonary squamous cell carcinoma [23].

However, also tumours where PD-L1 does not appear to be a prognostic marker [28], the use of the anti-PD1/PD-L1 drugs produces significant therapeutic effects [29]. In fact the only expression of the PD-L1 protein on tumor cells has been thought to be a good predictor of a patient's response.

In conclusion, in clinical practice, the most important purpose is to predict the treatment response from PD-1/PD-L1 blockade, by a correct standardization of operative protocols for the PD-L1 determination in tumor cells.

In our study, the absence of strong correlations between PD-L1 expression and survival of angiosarcoma patients could in part due to the small number of specimens, but also to the extreme aggressiveness of this tumor for which the prognosis is poor in the most of cases.

In angiosarcomas, despite PD-L1 is not related to the prognosis, its detection could be very useful in the therapeutic stratification of patients to enroll for specific therapies associated to PD-1/PD-L1 blockade.

\section{Abbreviations}

PD-1: Programmed cell death protein 1; PD-L1: Programmed death-ligand 1; IHC: immunohistochemistry; FFPE: Formalin-fixed, paraffin-embedded; H\&E: Hematossilin and eosin; OS: Overall survival; DFS: disease-free survival; TIL: Tumor infiltrating lymphocytes.

\section{Acknowledgements}

The authors thank all of the study participants for their great effort.

\section{Authors' contributions}

ADC and MC were responsible for the conception and design of the study. AP was responsible for provision of patients clinical information. GS and LM collected samples for immunohistochemical analysis. MDB, RDC and GB were responsible for immunohistochemical evaluation. All authors were involved in manuscript writing and provided final approval of the manuscript.

\section{Availability of data and materials}

All relevant data of the study were already included in the manuscript, including the survival curves although not significant.

\section{Ethics approval and consent}

This study was approved by the Ethics Committee of INT Fondazione Pascale.

Written informed consent was obtained from the patient for publication of this manuscript and any accompanying images. A copy of the written consent is available for review by the Editor-in-Chief of this journal.

\section{Competing Interests}

The authors have declared that no competing interest exists.

\section{References}

1. Young RJ, Brown NJ, Reed MW, et al. Angiosarcoma. Lancet Oncol. 2010; 11:983-91.

2. Costigan DC, Doyle LA. Advances in the Clinicopathologic and Molecular Classification of Cutaneous Mesenchymal Neoplasms. Histopathology. 2016; 68:776-95

3. Arora TK, Terracina KP, Soong J, et al. Primary and secondary angiosarcoma of the breast. Gland Surg. 2014; 3:28-34.

4. D'Angelo SP, Munhoz RR, Kuk D, et al. Outcomes of Systemic Therapy for Patients with Metastatic Angiosarcoma. Oncology. 2015; 89:205-14.

5. Ravo V, Falivene S, De Chiara A, et al. Treatment of cutaneous angiosarcoma of the face: efficacy of combined chemotherapy and radiotherapy. Tumori. 2013; 99:e211-5.

6. Ohguri T, Imada H, Nomoto S, et al. Angiosarcoma of the scalp treated with curative radiotherapy plus recombinant interleukin-2 immunotherapy. Int J Radiat Oncol Biol Phys. 2005; 61:1446-53.

7. Butte MJ, Peña-Cruz V, Kim MJ, et al. Interaction of human PD-L1 and B7-1. Mol Immunol. 2008; 45:3567-72.

8. Akbay EA, Koyama S, Carretero J, et al. Activation of the PD-1 pathway contributes to immune escape in EGFR-driven lung tumors. Cancer Discov. 2013; 3:1355-63. 
9. Zhang Y, Wang L, Li Y et al. Protein expression of programmed death 1 ligand 1 and ligand 2 independently predict poor prognosis in surgically resected lung adenocarcinoma. Onco Targets Ther. 2014; 7:567-73.

10. Choueiri TK, Fay AP, Gray KP et al. PD-L1 expression in non clear-cell renal cell carcinoma. Ann Oncol. 2014; 25:2178-84.

11. Taube JM, Klein A, Brahmer JR et al. Association of PD-1, PD-1 Ligands, and Other Features of the Tumor Immune Microenvironment with Response to Anti-PD-1 Therapy. Clin Cancer Res. 2014; 20:5064-74.

12. Harshman LC, Choueiri TK, Drake C. Subverting the B7-H1/PD-1 Pathway in Advanced Melanoma and Kidney Cancer. Cancer J. 2014; 20:272-80.

13. Soliman H, Khalil F, Antonia S. PD-L1 Expression Is Increased in a Subset of Basal Type Breast Cancer Cells. PLoS One. 2014; 9:e88557.

14. Topalian SL, Hodi FS, Brahmer JR. Safety, activity, and immune correlates of anti-PD-1 antibody in cancer. N Engl J Med. 2012; 366:2443-54.

15. Policarpio-Nicolas ML, Nicolas MM, Keh P, Laskin WB. Postradiation angiosarcoma of the small intestine: a case report and review of literature. Ann Diagn Pathol. 2006;10:301-5.

16. Ohaegbulam KC, Assal A, Lazar-Molnar E, et al. Human cancer immunotherapy with antibodies to the PD-1 and PD-L1 pathway. Trends Mol Med. 2015; 21: 24-33.

17. Schalper KA, Velcheti V, Carvajal D, et al. In situ tumor PD-L1 mRNA expression is associated with increased TILs and better outcome in breast carcinomas. Clin Cancer Res. 2014; 20:2773-82.

18. Chen YB, Mu CY, Huang JA. Clinical significance of programmed death-1 ligand-1 expression in patients with non-small cell lung cancer: a 5-year-follow-up study. Tumori. 2012; 98 :751-5.

19. Wang L, Ren F, Wang Q, et al. Significance of Programmed Death Ligand 1 (PD-L1) Immunohistochemical Expression in Colorectal Cancer. Mol Diagn Ther. 2016;20:175-81.

20. Jacquelot N, Roberti MP, Enot DP, et al. Immunophenotyping of stage III melanoma reveals parameters associated with patient prognosis. J Invest Dermatol. 2016; 136:994-1.

21. $\mathrm{Xu} \mathrm{F}, \mathrm{Xu} \mathrm{L}$, Wang $\mathrm{Q}$, et al. Clinicopathological and prognostic value of programmed death ligand-1 (PD-L1) in renal cell carcinoma: a meta-analysis. Int J Clin Exp Med. 2015; 8:14595-603.

22. Lin C, Chen X, Li M, et al. Programmed Death-Ligand 1 Expression Predicts Tyrosine Kinase Inhibitor Response and Better Prognosis in a Cohort of Patients With Epidermal Growth Factor Receptor Mutation-Positive Lung Adenocarcinoma. Clin Lung Cancer. 2015; 16:e25-35.

23. Yang $\mathrm{CY}$, Lin MW, Chang YL, et al. Programmed cell death-ligand 1 expression is associated with a favourable immune microenvironment and better overall survival in stage I pulmonary squamous cell carcinoma. Eur J Cancer. 2016; 57:91-103.

24. Muenst S, Schaerli AR, Gao F, et al. Expression of programmed death ligand 1 (PD-L1) is associated with poor prognosis in human breast cancer. Breast Cancer Res Treat. 2014; 146:15-24.

25. D'Angelo SP, Shoushtari AN, Agaram NP, et al. Prevalence of tumor-infiltrating lymphocytes and PD-L1 expression in the soft tissue sarcoma microenvironment. Hum Pathol. 2015; 46:357-65.

26. Leite KR, Reis ST, Junior JP, et al. PD-L1 expression in renal cell carcinoma clear cell type is related to unfavorable prognosis. Diagn Pathol. 2015; 10:189.

27. Muenst S, Soysal SD, Gao F, et al. The presence of programmed death 1 (PD-1)-positive tumor-infiltrating lymphocytes is associated with poor prognosis in human breast cancer. Breast Cancer Res Treat. 2013; 139:667-76.

28. Ukpo OC, Thorstad WL, Lewis JS. B7-H1 expression model for immune evasion in human papillomavirus-related oropharyngeal squamous cell carcinoma. Head Neck Pathol. 2013; 7:113-21.

29. Sorensen SF, Zhou W, Dolled-Filhart M, et al. PD-L1 Expression and Survival among Patients with Advanced Non-Small Cell Lung Cancer Treated with Chemotherapy. Translational Oncology. 2016, 9:64-69. 